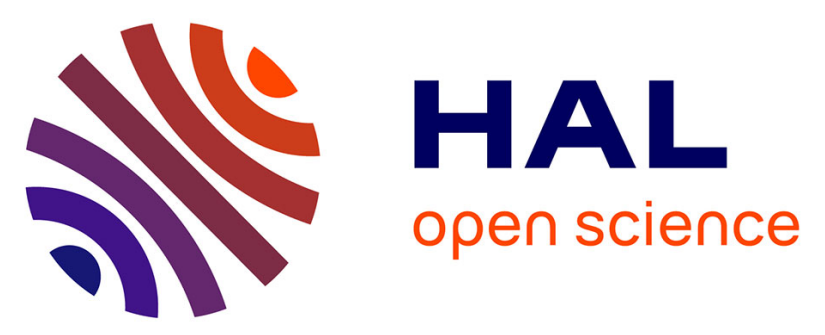

\title{
Évolution des comportements et indicateurs de santé mentale entre 2006 et 2010 dans la population au travail en France
}

L. Malard, J.-F. Chastang, Isabelle Niedhammer

\section{- To cite this version:}

L. Malard, J.-F. Chastang, Isabelle Niedhammer. Évolution des comportements et indicateurs de santé mentale entre 2006 et 2010 dans la population au travail en France. Epidemiology and Public Health $=$ Revue d'Epidémiologie et de Santé Publique, 2017, 65 (4), pp.309-320. 10.1016/j.respe.2017.04.056 . hal-01548148

\section{HAL Id: hal-01548148 \\ https: / hal.sorbonne-universite.fr/hal-01548148}

Submitted on 27 Jun 2017

HAL is a multi-disciplinary open access archive for the deposit and dissemination of scientific research documents, whether they are published or not. The documents may come from teaching and research institutions in France or abroad, or from public or private research centers.
L'archive ouverte pluridisciplinaire HAL, est destinée au dépôt et à la diffusion de documents scientifiques de niveau recherche, publiés ou non, émanant des établissements d'enseignement et de recherche français ou étrangers, des laboratoires publics ou privés. 
Evolution des comportements et indicateurs de santé mentale entre 2006 et 2010 dans la population au travail en France

Changes in behaviours and indicators of mental health between 2006 and 2010 in the French working population

L Malard (1,2,3), JF Chastang (1,2), I Niedhammer (1,2)

(1) INSERM, UMR_S 1136, Institut Pierre Louis d'Epidémiologie et de Santé Publique, Equipe de Recherche en Epidémiologie Sociale, F-75013, Paris, France

(2) Sorbonne Universités, UPMC Univ Paris 06, UMR S 1136, Institut Pierre Louis d'Epidémiologie et de Santé Publique, Equipe de Recherche en Epidémiologie Sociale, F75013, Paris, France

(3) Université de Versailles St-Quentin, France

Correspondance :

Dr. Isabelle Niedhammer

INSERM U1136 - Equipe ERES

Faculté de Médecine de Saint-Antoine

27 rue Chaligny

75012 Paris

France

Email: isabelle.niedhammer@inserm.fr

Titre courant : Santé mentale et crise économique 


\begin{abstract}
Background

The 2008 economic crisis could have repercussions on mental health but the studies on this topic are sparse, in particular among the working population. However, mental health at work is a crucial issue involving substantial costs and consequences. The aim of the study was to assess the changes in behaviours and indicators of mental health in the French working population between 2006 and 2010, and to explore the differential changes according to age, origin, occupation, activity sector, public/private sector, selfemployed/employee status and work contract.
\end{abstract}

\title{
Methods
}

The data came from the prospective national representative Santé et Itinéraire Professionnel (SIP) survey, including a sample of 5600 French workers interviewed in 2006 and 2010. The behaviours and indicators of mental health studied were excessive alcohol consumption, smoking, sleep problems (sleep troubles and/or insufficient sleep duration), psychotropic drug use (antidepressants, anxiolytics and/or hypnotics), and poor self-reported health. Generalized estimating equations were used to analyze the changes in behaviours and indicators of mental health, and the analyses were adjusted for age. Covariates (age, origin, occupation, activity sector, public/private sector, selfemployed/employee status and type of contract) were added separately to assess differential changes.

\section{Results}

Increases in excessive alcohol consumption among women, sleep problems among men, and smoking, insufficient sleep duration and poor self-reported health for both genders 
were observed in the French working population between 2006 and 2010. Some differential changes were observed, negative changes being more likely to affect young workers and workers with permanent contract.

\section{Conclusion}

Prevention policies should consider that there may be a deterioration in behaviours and indicators of mental health in times of economic crisis, especially among some sub-groups of the working population such as young workers and workers with permanent contract. These changes might foreshadow a forthcoming increase in mental disorders.

Key words: Economic recession. Mental health. Workplace. France.

\section{Résumé}

\section{Position du problème}

Les répercussions de la crise économique de 2008 sur la santé mentale de la population sont encore mal connues, en particulier dans la population au travail. Pourtant la santé mentale au travail est un enjeu important par ses coûts et conséquences substantiels. L'objectif était d'évaluer l'évolution de la prévalence de comportements et indicateurs de santé mentale dans la population au travail en France entre 2006 et 2010, et d'étudier les évolutions différentielles selon l'âge, l'origine, la profession, le secteur d'activité, le secteur public/privé, le statut indépendant/salarié et le type de contrat.

\section{Méthodes}

L'enquête Santé et itinéraire Professionnel (SIP) est une enquête prospective représentative de la population générale française, et parmi les individus interrogés, 5600 étaient en 
emploi en 2006 et en 2010. Les comportements et indicateurs de santé mentale étudiés étaient la consommation d'alcool à risque, le tabagisme, les problèmes du sommeil (troubles du sommeil et/ou durée de sommeil insuffisante), la prise de psychotropes (antidépresseurs, anxiolytiques et/ou hypnotiques), et la mauvaise santé perçue. Des équations d'estimation généralisées ont été utilisées pour étudier les évolutions, les analyses étaient ajustées sur l'âge, puis des covariables (âge, origine, profession, secteur d'activité, secteur public/privé, statut indépendant/salarié et type de contrat) ont été introduites séparément pour évaluer les évolutions différentielles.

\section{Résultats}

Dans la population au travail en France, des augmentations de la prévalence de la consommation d'alcool à risque chez les femmes, des problèmes de sommeil chez les hommes, et de tabagisme, de la durée de sommeil insuffisante et de la mauvaise santé perçue pour les deux genres ont été observées entre 2006 et 2010. Quelques évolutions différentielles ont été mises en évidence, en particulier au détriment des plus jeunes et des personnes en contrat permanent.

\section{Conclusion}

En conclusion, les politiques de prévention devraient considérer que les prévalences des comportements et indicateurs de mauvaise santé mentale peuvent avoir augmenté en période de crise économique, en particulier pour certains sous-groupes de la population au travail tels que les plus jeunes et les personnes en contrat permanent. Ces évolutions pourraient laisser présager une augmentation future des pathologies mentales.

Mots-clés : Crise économique. Santé mentale. Population au travail. France. 


\section{Introduction}

La crise économique de 2008 est une des plus sévères grandes crises économiques depuis un siècle, elle a particulièrement touché les pays développés dont la France. Une crise économique se traduit par un ralentissement de l'activité économique entrainant des faillites, une hausse du chômage, mais aussi par des répercussions sur les salaires, et des tensions sociales et politiques. En France, le taux de croissance a décliné de 2,66\% en 2009 par rapport à 2008 et le taux de chômage est passé de 7,3\% en 2008 à 9,3\% en 2010. La dégradation de ces indicateurs bien qu'importante était moins forte qu'en Grèce, en Espagne ou encore en Irlande qui ont été parmi les plus touchés par la crise économique. En 2010, le taux de chômage avait triplé par rapport à 2008 en Irlande et en Espagne, jusqu'à atteindre 20\% en 2010 en Espagne. En Grèce, l'augmentation rapide du chômage s'est plutôt faite après 2010. Au contraire de la France et de la plupart des pays européens, la Pologne n'est jamais entrée en récession et a connu une augmentation modérée de son taux de chômage, du même ordre que celle de la France.

Dans un rapport de 2009, l'Organisation Mondiale de la Santé (OMS) s'est inquiétée des répercussions que pourrait avoir la crise économique sur la santé mentale et physique, du fait de son impact sur les déterminants de la santé tels que le revenu, l'emploi, l'éducation, et l'alimentation, déclarant que les populations vulnérables devraient être protégées à cause des inégalités de santé [1]. En France, un rapport récent du HCSP montre que les crises économiques et les politiques qu'elles suscitent ont des conséquences sur la santé et les inégalités sociales de santé et des impacts complexes à court terme [2].

Différentes hypothèses existent sur l'effet de la crise économique sur la santé [3-5]. D’une part, certains auteurs pensent que les périodes de crise économique sont bénéfiques pour la santé des populations. En effet, le temps pour des loisirs comme l'activité sportive 
augmente ce qui est bénéfique pour la santé. Les expositions aux conditions de travail à risque pour la santé (conditions psychosociales de travail, expositions physiques, accidents du travail, etc.) diminuent puisque l'activité économique diminue. Enfin le pouvoir d'achat diminue laissant moins de moyens pour la consommation d'alcool et les déplacements en voiture donc il y a de la consommation d'alcool et des accidents de la route.

D'autre part, des auteurs ont fait l'hypothèse qu'une période de récession entraine des effets négatifs sur la santé des populations ; il peut y avoir une perte de statut du fait d'une perte d'emploi, ou d'un niveau de rémunération inférieur à la normale, cette perte de statut signifie aussi une perte de l'estime de soi, d'expression de soi, de confiance en soi et d'accès à des réseaux sociaux. Le stress psychosocial associé à cette perte peut également avoir des dommages, et le fait d'essayer de soulager la détresse psychologique par l'alcool, les drogues légales et illégales, le tabac ou la sous- ou suralimentation fait augmenter la morbidité et ajoute de nouveaux problèmes de santé [6].

Des études se sont intéressées à l'évolution de la santé mentale en période de récession en population générale, montrant notamment une augmentation du taux de suicide [7-19], de la prévalence de pathologies mentales telles que les troubles de l'humeur, anxieux, somatoformes, liés à l'alcool, et dépressifs [18,20-25], tandis que d'autres études n'ont montré aucune évolution voire une diminution du taux de suicide $[8,26]$, et de la prévalence des idées et tentatives de suicide [27]. Ces augmentations étaient associées avec des augmentations du taux de chômage, de l'insécurité de l'emploi, des difficultés financières, des difficultés de remboursement de prêt, des faillites ou encore des événements de travail indésirables [7-11,16,18,19,21,23,25,27,28]. Il a également été montré que ces effets de la crise sur la santé pouvaient être atténués avec des programmes actifs de marché du travail [18,29,30], et que, au contraire, les politiques d'austérité et les coupes budgétaires dans le domaine de la santé étaient en partie responsables de ces 
augmentations des problèmes de santé $[18,22,29]$. Cependant, des comportements et indicateurs de santé mentale pourraient être plus sensibles au changement à plus court terme et être des marqueurs précoces d'une possible augmentation des pathologies mentales à plus long terme.

Les études sur l'évolution de comportements et indicateurs de santé avaient des résultats mitigés. En effet, en période de récession, ces études ont montré en population générale une augmentation de la consommation d'alcool [28,31-34], de la mauvaise santé perçue [35-37], mais aussi une diminution de la consommation d'alcool [38,39], de la consommation de tabac, une amélioration du temps de sommeil [40], et une stabilisation de la prévalence de mauvaise santé perçue [41].

La littérature est éparse dans la population au travail. Pourtant, la santé mentale au travail est un sujet crucial impliquant des coûts et conséquences importants $[42,43]$, et nous pouvons supposer que la crise économique pourrait contribuer à augmenter les problèmes de santé mentale aussi dans la population au travail notamment par le biais d'une dégradation des conditions psychosociales de travail. Or, nous avons précédemment montré que les conditions psychosociales de travail se sont dégradées durant la crise économique de 2008 en Europe, en particulier pour les pays les plus touchés par la crise comme la Grèce ou l'Irlande [44]. En France entre 2006 et 2010, nous avons également mis en évidence que la latitude décisionnelle s'est dégradée, de plus nous avons aussi observé une augmentation de la forte demande psychologique (chez les femmes seulement), du faible soutien social, des faibles récompenses, et parmi les facteurs émergents, de la forte demande émotionnelle (chez les femmes seulement), de la qualité empêchée, du conflit éthique (chez les femmes seulement), des tensions avec le public (chez les femmes seulement), et du déséquilibre travail-famille [45]. 
A notre connaissance, seules deux études se sont intéressées à l'évolution des comportements et indicateurs de santé mentale en population au travail. Une étude a montré une diminution de la durée de sommeil, et une augmentation de la somnolence perçue, de l'impression de suffocation, des cauchemars et de la transpiration nocturne entre 2005 et 2010 en Grèce [46]. La seconde étude a été réalisée en Suède lors de la récession du début des années 90 dans les pays nordiques, il a été observé une augmentation de la prévalence de la détresse psychologique et de la mauvaise santé perçue entre 1991 et 2000 [47].

En France, une de nos précédentes études s'est intéressée à l'évolution de pathologies mentales, l'épisode dépressif majeur $(E D M)$ et le trouble d'anxiété généralisé (TAG), à l'aide d'un entretien diagnostique [48]. Cependant, aucune évolution de la prévalence d'EDM et de TAG n'a été mise en évidence entre 2006 et 2010 dans la population au travail en France. Le délai entre le début de la crise économique et la possible apparition de pathologies mentales n'est pas connu, l'évaluation de l'EDM et du TAG en 2010 était peut-être trop précoce pour observer un impact de la crise économique sur ces pathologies. C'est pourquoi il nous a paru intéressant d'évaluer dans la même population d'étude l'évolution de comportements et indicateurs de santé mentale comme marqueurs précoces d'une possible évolution à venir des pathologies mentales.

De plus, la segmentation du marché du travail oppose le marché primaire où les emplois sont stables, le niveau de compétence est plutôt élevé, la rémunération l'est aussi, et des perspectives de promotion existent, au marché secondaire où les emplois sont précaires, les rémunérations basses, la mobilité forte et les perspectives de promotion sont faibles [4951]. Certains sous-groupes de la population sont plus susceptibles d'avoir un emploi dans le marché secondaire comme les femmes, les plus jeunes, les travailleurs les moins qualifiés ou encore les personnes d'origine étrangère [52,53]. Il nous paraissait intéressant 
d'étudier si les évolutions étaient différentes pour ces sous-groupes qui caractérisent aussi le marché secondaire. En outre, ces mêmes sous-groupes ont été plus durement touchés par la crise économique, comme les plus jeunes, et les professions les moins qualifiées, des secteurs d'activités comme celui de la construction [54,55].

Dans les hypothèses faites sur les conséquences de la crise économique sur la santé, il y a une potentielle augmentation de la consommation d'alcool afin de soulager le stress psychosocial engendré par la crise. La consommation d'alcool et le tabagisme étant très associé, il a été choisi d'étudier l'évolution de ces deux comportements. Les troubles du sommeil sont classés dans les troubles mentaux et du comportement (Chapitre V de la CIM-10), ils peuvent être des symptômes de dépression et d'anxiété. Les pathologies mentales sont notamment traitées par des médicaments psychotropes comme les antidépresseurs, les anxiolytiques ou encore les hypnotiques, c'est pourquoi l'évaluation de la santé mentale peut être appréhendée par la prise de ces psychotropes. Il s'agit donc d'un marqueur indirect de ces pathologies. Enfin, la santé perçue fait référence à la perception qu'a un individu de sa santé générale, elle englobe donc tous les aspects de la santé à savoir l'absence de maladie mais aussi un bien-être physique, mental et social. La santé perçue est un bon prédicteur de morbidité et de mortalité future [56-58] et est associée à la santé mentale [59-61]. C'est pourquoi tous ces comportements et indicateurs de santé mentale ont été choisis.

L'objectif de cette étude était donc d'évaluer, à l'aide d'une enquête nationale prospective, l'évolution de comportements et indicateurs de santé mentale, tels que la consommation d'alcool à risque, le tabagisme, les problèmes de sommeil, la prise de psychotropes, et la mauvaise santé perçue entre 2006 et 2010 dans la population au travail en France. Et, dans un deuxième temps, l'objectif était d'étudier les évolutions différentielles de ces comportements et indicateurs de santé mentale selon des sous-groupes de population au 
travail, c'est-à-dire en fonction de l'âge, l'origine, la profession, le secteur d'activité, le secteur public/privé, le type de contrat et le statut indépendant/salarié.

\section{Matériel et méthodes}

\section{Population}

L'enquête nationale prospective et représentative SIP a été dirigée par les Ministères du Travail et de la Santé (Direction de l'Animation de la Recherche, des Etudes et des Statistiques (DARES) et Direction de la Recherche, des Etudes, de l'Evaluation et des Statistiques (DREES)), le Centre d'Etudes de l'Emploi (CEE) et l'Institut National de la Statistique et des Etudes Economiques (INSEE). Cette enquête a été mise en place afin d'explorer les liens complexes qui existent entre santé et travail [62]. Plusieurs articles ont été publiés sur cette enquête par notre équipe [45,48,63-68].

En 2006, des ménages ont été sélectionnés aléatoirement parmi la base des ménages du recensement de 1999, enrichie des nouveaux logements de 1999 à 2006, puis dans les ménages sélectionnés, un individu âgé de 20 à 74 ans a été choisi pour être interrogé en prenant celui qui avait le prénom qui arrivait en premier dans l'ordre alphabétique. Finalement, 13648 hommes et femmes de la population générale ont été interrogés à propos de leur itinéraire professionnel et de leur santé par un enquêteur en face à face au domicile de l'enquêté. Le taux de réponse à l'enquête en 2006 était de 76\%. Quatre années plus tard, ces personnes ont été recontactées afin de participer à la seconde vague de collecte de données, 11016 individus ont pu être réinterrogés, soit environ $81 \%$ de l'échantillon initial.

Parmi les individus interrogés, 8107 déclaraient être en emploi en 2006, parmi lesquels 51 sont décédés, 1383 n'ont pas pu être réinterrogés en 2010, 277 étaient au chômage et 796 ont pris leur retraite ou ne travaillaient pas pour d'autres raisons. Au final, 5600 individus 
ayant répondu aux deux vagues de l'enquête travaillaient en 2006 et en 2010 et constituaient notre échantillon d'étude.

\section{Les comportements et indicateurs de santé mentale}

\section{Le tabagisme et la consommation d'alcool à risque}

Le tabagisme était évalué à partir de la question «En ce moment, est-ce que vous fumez? », les individus ayant répondu positivement à la question, même si la consommation n'était pas journalière, étaient considérés comme fumeurs. Les autres individus (non-fumeurs et anciens fumeurs) étaient considérés comme non-fumeurs.

A partir de trois questions, sur la fréquence de consommation d'alcool, le nombre de verres consommés par jour et la fréquence de consommation excessive d'alcool (au moins six verres d'alcool consommés au cours d'une même occasion), des profils de consommation ont été définis. La consommation d'alcool à risque était définie par une consommation excessive d'alcool une fois par mois ou plus, ou une consommation de plus de 21 verres d'alcool pour les hommes (14 verres pour les femmes) par semaine.

\section{Le sommeil}

Trois variables relatives au sommeil ont été étudiées, les troubles du sommeil, la durée de sommeil insuffisante, et les problèmes de sommeil (troubles du sommeil et/ou durée de sommeil insuffisante).

Les troubles du sommeil ont été définis à l'aide de la question «Au cours des douze derniers mois, avez-vous ressenti des troubles du sommeil (difficultés à s'endormir, réveils la nuit, éveil précoce sans réussir à se rendormir, ...) ? », les individus qui répondaient «plusieurs fois par semaine » ou «presque tous les jours » étaient considérés comme ayant des troubles du sommeil. 
Quant à la durée de sommeil insuffisante, à la question «Au cours des douze derniers mois, votre nombre d'heures de sommeil la nuit était-il... », les individus ayant répondu « insuffisant plusieurs fois par semaine » ou «insuffisant presque tous les jours » étaient classés comme ayant une durée de sommeil insuffisante.

Les problèmes de sommeil étaient une variable agrégée des deux premières variables et correspondaient à des troubles du sommeil et/ou une durée de sommeil insuffisante comme définis précédemment.

Les critères de fréquence utilisés pour définir les cas ont suivi les recommandations du DSM-V, qui définit l'insomnie par des difficultés qui surviennent au moins trois nuits par semaine.

\section{La consommation de psychotropes}

Le questionnaire SIP comprenait trois questions sur la prise de psychotropes au cours des 12 derniers mois. Ces questions étaient basées sur trois listes de médicaments présentées à la personne interrogée.

La première liste concernait la prise d'antidépresseurs, la variable était déjà dichotomique, les cas étant définis par les consommateurs d'antidépresseurs, quelle que soit la fréquence d'usage.

La seconde liste concernait la prise d'hypnotiques. La variable a été dichotomisée, les individus prenant des hypnotiques «une ou plusieurs fois par semaine» ou «tous les jours » étaient classés comme utilisateurs d'hypnotiques, les autres ayant répondu « jamais ou rarement » ou «une ou plusieurs fois par mois » étaient considérés comme nonutilisateurs d'hypnotiques. 
Enfin, la troisième liste concernait la prise d'anxiolytiques. La variable a été dichotomisée de la même manière que pour les hypnotiques, à savoir les individus ayant répondu en utiliser au moins une fois par semaine étaient considérés comme utilisateurs d'anxiolytiques, les autres comme non-utilisateurs.

La prise de psychotropes, correspondant à la prise d'antidépresseurs et/ou d'hypnotiques et/ou d'anxiolytiques telles que définies précédemment, a également été étudiée.

\section{La santé perçue}

Le questionnaire SIP contenait la question «Comment est votre état de santé général ? », avec comme réponses possibles : «très bon », «bon », «moyen », «mauvais », « très mauvais ». D'après cette question, une variable binaire a été créée permettant de distinguer les individus ayant une mauvaise santé perçue (comprenant ceux ayant répondu moyen, mauvais ou très mauvais) et les individus ayant une (très) bonne santé perçue.

\section{Les covariables}

Les covariables étaient l'âge, l'origine (française ou étrangère), la profession (cadres, professions intermédiaires, employés et ouvriers), le secteur d'activité (agriculture et pêche, industrie, construction, et services), le secteur public/privé, le type de contrat (précaire ou stable) et le statut indépendant/salarié. La profession était codée selon la classification des professions et catégories socioprofessionnelles françaises (PCS-INSEE), quant au secteur d'activité, il a été codé d'après la Nomenclature d'Activités Française (NAF).

\section{La pondération}

Pour être représentatif de la population française au travail de 2006, des pondérations ont été calculées en utilisant le calage sur marges et les probabilités inverses afin de réduire les 
biais dus à la non-réponse en 2006 et à l'attrition en 2010 [69]. Tout d'abord, un calage sur marges a été réalisé avec l'échantillon de 2006 sur les variables âge, statut de l'emploi (en emploi/au chômage/non-actif) × âge, aire urbaine, taille du ménage, activité (nomenclature d'activité française). Puis des groupes de réponse homogènes ont été formés d'après les caractéristiques de 2006 (statut de l'emploi, aire urbaine, niveau d'éducation, genre et santé perçue), et la probabilité de répondre à l'enquête en 2010 a été calculée pour chacun des groupes. Les pondérations calculées par calage sur marges (pour la non-réponse en 2006) ont été multipliées par l'inverse de la probabilité de réponse en 2010. Enfin, avec les données pondérées précédentes, un second calage sur marges sur l'unité territoriale, l'aire urbaine, l'âge $\times$ genre, le niveau d'éducation, la nationalité et la taille du ménage a été réalisé sur l'échantillon des individus interrogés en 2006 et 2010 afin d'être représentatif de la population générale de 2006. Les marges ont été calculées à partir des quatre enquêtes Emploi trimestrielles de 2006. Les pondérations ont été inclues dans toutes les analyses statistiques.

\section{Les analyses statistiques}

Pour évaluer l'évolution des prévalences des comportements et indicateurs de santé mentale, des équations d'estimation généralisées ont été utilisées avec une fonction de lien logit $[70,71]$. L'avantage de ces modèles était qu'ils prenaient en compte la corrélation entre les deux mesures d'un même individu. La variable dépendante était le comportement ou l'indicateur de santé mentale, et une variable binaire représentant l'année $(0=2006$ et 1=2010) était la variable d'intérêt. Les modèles étaient ajustés sur l'âge (variable continue).

Pour tester si les évolutions étaient différentes selon les covariables (âge, origine, profession, secteur d'activité, secteur public/privé, statut indépendant/salarié et type de contrat), chaque covariable et les termes d'interaction entre la covariable et l'année ont été 
ajoutés et testés successivement. Cette analyse a été effectuée pour tous les comportements et indicateurs de santé mentale, y compris sur ceux où l'effet global était non significatif. Tous les modèles étaient ajustés sur l'âge.

Les analyses statistiques ont été stratifiées sur le genre et réalisées sous SAS 9.3 (SAS Institute Inc, Cary, NC).

\section{Résultats}

L'échantillon d'étude était composé de 2679 hommes et 2921 femmes en emploi en 2006 et qui étaient toujours en emploi en 2010 (Tableau 1). De façon très marquée, le nombre de contrats temporaires a diminué d'environ 50\% pour les deux genres, en effet, en 2006 il y avait $12 \%$ et $16 \%$ d'hommes et de femmes en contrat temporaire alors qu'en 2010 , ils n'étaient plus que 5\% et $8 \%$ dans cette situation. En 2006 et 2010, des différences de genre ont été observées. Les hommes étaient plus fréquemment ouvriers et cadres, tandis que les femmes étaient plus fréquemment employées. Elles travaillaient plus fréquemment dans le secteur public, dans le secteur des services et avec des contrats temporaires alors que les hommes étaient plus fréquemment indépendants. Les prévalences de mauvais comportements de santé, tabagisme et consommation d'alcool à risque, étaient plus élevées chez les hommes que chez les femmes en 2006 et en 2010. Par contre, les prévalences des indicateurs de mauvaise santé (mauvaise santé perçue, problèmes de sommeil, troubles du sommeil et durée de sommeil insuffisante) étaient plus élevées parmi les femmes pour les deux années, ainsi que les prévalences de prise de psychotropes, pour les trois classes thérapeutiques, les antidépresseurs, les hypnotiques ou les anxiolytiques.

Après ajustement sur l'âge, des augmentations des prévalences du tabagisme pour les deux genres, de consommation d'alcool à risque pour les femmes, des problèmes de sommeil parmi les hommes, de la durée de sommeil insuffisante pour les deux genres et de la 
mauvaise santé perçue pour les deux genres ont été observées entre 2006 et 2010 (Tableau 2). Aucune évolution des prévalences des troubles du sommeil, de consommation de psychotropes, d'antidépresseurs, d'hypnotiques ou d'anxiolytiques n'a été mise en évidence entre 2006 et 2010.

Des évolutions différentielles des comportements et indicateurs de santé mentale selon l'âge, l'origine, la profession, le secteur d'activité, le secteur public/privé, le statut indépendant/salarié et le type de contrat ont été mises en évidence (Tableau 3).

En effet, l'évolution de la consommation d'alcool à risque était significativement différente selon la profession chez les femmes : parmi les cadres et les ouvrières, aucune évolution n'a été montrée, alors qu'il y avait une augmentation de la prévalence de consommation d'alcool à risque pour les professions intermédiaires et les employées.

L'augmentation de la prévalence des problèmes de sommeil ne concernait que les plus jeunes hommes (20-40 ans), les travailleuses d'origine étrangère et les hommes en contrat permanent.

Entre 2006 et 2010, la prévalence des troubles du sommeil n’a significativement augmenté que pour les femmes d'origine étrangère, alors qu'il n'y avait pas d'évolution pour les femmes d'origine française.

Pour la durée de sommeil insuffisante, la prévalence chez les hommes n'a augmenté entre 2006 et 2010 que pour les 20-40 ans et les hommes en contrat permanent, tandis qu'elle a significativement diminué pour les hommes en contrat temporaire.

Des évolutions différentielles ont été mises en évidence pour la prise de psychotropes et d'antidépresseurs selon le secteur public/privé. Les évolutions de la prévalence de la prise de psychotropes et d'antidépresseurs étaient différentes selon le secteur public ou privé. 
Même si, pour chacun des deux sous-groupes, les évolutions n'étaient pas significatives, la prévalence de la prise de psychotropes et d'antidépresseurs montrait une tendance à l'augmentation entre 2006 et 2010 dans le secteur public, alors que la tendance était plutôt à la diminution dans le secteur privé.

Enfin, la prévalence de la mauvaise santé perçue a augmenté parmi les hommes de 30-40 ans et parmi les hommes de plus de 50 ans, ainsi que chez les femmes ayant un contrat permanent, tandis que pour les autres groupes d'âge chez les hommes et les femmes en contrat temporaire, aucune évolution significative n'a été mise en évidence.

\section{Discussion}

Les comportements et indicateurs de santé mentale ont montré une dégradation, avec une augmentation de la consommation d'alcool à risque chez les femmes, des problèmes de sommeil chez les hommes, du tabagisme, de la durée de sommeil insuffisante et de la mauvaise santé perçue pour les deux genres dans la population au travail entre 2006 et 2010. Néanmoins, aucune évolution de la prise de psychotropes (antidépresseurs, hypnotiques et anxiolytiques) n'a été observée. Quelques évolutions différentielles ont été mises en évidence, en particulier au détriment des plus jeunes et des personnes en contrat permanent.

La période examinée était intéressante du fait de l'apparition de la crise économique en 2008, qui pourrait expliquer en partie nos résultats. Cependant, nos analyses n'ont pas permis d'attribuer les évolutions négatives des comportements et indicateurs de santé mentale exclusivement à la crise économique de 2008 qui s'est produite au milieu de la période 2006-2010. Pour montrer un potentiel effet de la crise économique, une des solutions serait d'utiliser une approche quasi-expérimentale, en choisissant comme référence soit la période antérieure à la crise soit un pays/région n'ayant pas subi la crise 
économique, comme certaines études l'ont fait en prenant comme référence la Pologne $[36,72,73]$. Ce genre d'approche s'apparente à une approche expérimentale dans laquelle l'expérience est naturelle et ne peut être contrôlée. Cependant, ces modèles ont également leurs limites du fait de la non-randomisation des groupes rendant les analyses statistiques plus délicates, notamment dans le choix des variables d'ajustement [74].

Néanmoins, ces résultats semblaient cohérents avec ce qui peut être attendu dans une telle période de déclin de l'activité économique, de hausse du chômage, de diminution du pouvoir d'achat, de pessimisme ambiant, etc. Mais, des tendances à l'augmentation de certaines pratiques, comme le binge drinking, compliquent l'interprétation des résultats. On peut par exemple penser que l'augmentation de la consommation d'alcool à risque a été influencée par l'augmentation du binge drinking observable depuis quelques années, il est donc difficile de dire que cette augmentation observée est due à la crise économique. Les évolutions différentielles observées étaient également cohérentes avec l'hypothèse que certains sous-groupes de population plus touchés par la crise économique, tels que les plus jeunes travailleurs, puissent également être les plus touchés par une dégradation de la santé mentale. Cependant, les résultats sur l'augmentation de la prévalence des problèmes de sommeil, de la durée de sommeil insuffisante et de la mauvaise santé perçue observés seulement pour les individus en contrat permanent étaient difficiles à interpréter. Ceci peut éventuellement s'interpréter comme des conditions de travail plus difficiles qui ont un impact sur les travailleurs en contrat permanent qui devaient supporter ces conditions sans rien dire de peur de perdre leur emploi et/ou de peur de ne pas pouvoir trouver un autre emploi. Tandis que les travailleurs en contrat temporaire savaient que ces conditions allaient changer dès lors qu'ils changeraient d'entreprise. 


\title{
Comparaison avec la littérature
}

Dans la littérature, peu d'études se sont intéressées à l'évolution des comportements et indicateurs de santé mentale en population au travail. C'est pourquoi, afin d'avoir un point de comparaison avec la littérature, nous avons réalisé des analyses complémentaires en population générale. Les résultats en population générale étaient plutôt stables par rapport aux résultats en population au travail.

\begin{abstract}
Alcool et tabac
A notre connaissance, aucune étude sur l'évolution des consommations de tabac et d'alcool en période de crise économique n'a été réalisée dans la population au travail. En population générale, des études ont évalué l'évolution de la consommation d'alcool et de tabac en période de crise économique, que ce soit celle de 2008 ou des crises économiques antérieures.
\end{abstract}

Nos résultats ont montré une augmentation du tabagisme pour les deux genres et de la consommation d'alcool à risque seulement pour les femmes entre 2006 et 2010 dans la population au travail et ces résultats ont aussi été trouvés dans la population générale en France. Comme dans notre étude, les chiffres du baromètre santé ont montré une augmentation de la prévalence du tabagisme chez les femmes en population générale et également chez les actifs occupés mais cette augmentation n'était pas significative chez les hommes en population générale entre 2005 et 2010 [75]. Il a également été observé une augmentation de la consommation d'alcool à risque chez les femmes en population générale entre 2005 et 2010 [76]. En Espagne, il a été observé une augmentation des troubles liés à la consommation d'alcool, mesurés à l'aide d'un entretien diagnostique [21], ce qui concordait avec nos résultats. D'autres études étaient en accord avec nos résultats montrant une augmentation de la consommation d'alcool [18,21,28,31-34,77-79], tandis 
que des études observaient des résultats discordants des nôtres car elles montraient une diminution de la consommation de tabac [40] et d'alcool [38,39].

\section{Le sommeil}

Dans la population au travail et aussi dans la population générale des données de l'enquête SIP, nous n'avons trouvé aucune évolution de la prévalence des troubles du sommeil et une augmentation des problèmes de sommeil, qui était surtout le fait de l'augmentation de la prévalence de la durée de sommeil insuffisante, entre 2006 et 2010 en France. A notre connaissance, une seule étude antérieure a été réalisée dans la population au travail. Dans cette étude transversale périodique parmi des hommes salariés de l'Organisation Nationale des Chemins de Fer en Grèce, il a été montré qu'entre 2005 et 2010 la durée de sommeil a diminué, la somnolence diurne a augmenté, ainsi que la somnolence perçue, l'impression de suffocation, les cauchemars et les sueurs nocturnes [46], ce qui concordait avec nos résultats sur la durée de sommeil insuffisante.

D'autres études ont également examiné l'évolution du sommeil en population générale, les résultats étaient mitigés. Une étude a montré une amélioration du temps de sommeil en Islande entre 2007 et 2009 [40], en désaccord avec nos résultats. Cependant, une augmentation de la perte de sommeil liée à des inquiétudes a été observée en Angleterre au début des années 90 où l'Angleterre a subi un déclin économique [80], ce qui concordait avec nos résultats sur la durée de sommeil insuffisante. De même, une crise économique est survenue en Finlande au début des années 90 et une étude a montré que les prévalences de l'insomnie chez les femmes et des ronflements chez les hommes avaient augmenté entre 1983-1987 et 1992-1995 dans le sud de la Finlande, mais que les autres variables relatives au sommeil - trouble de somnolence excessive, fatigue, parasomnie - étaient restées stables [78], ce qui était en accord avec nos résultats en population générale sur la durée de sommeil insuffisante. 


\section{La prise de psychotropes}

Aucune évolution de la prise de psychotropes (antidépresseurs, anxiolytiques et hypnotiques) n'a été mise en évidence dans nos résultats entre 2006 et 2010 dans la population au travail en France. En désaccord avec nos résultats, une étude prospective parmi des travailleurs d'une entreprise de production d'aluminium aux Etats-Unis a montré que les visites à l'hôpital et ambulatoires, et l'approvisionnement annuel en médicaments liés à la santé mentale ont augmenté pour tous les travailleurs après 2009 et que l'amplitude de l'augmentation de l'utilisation de médicaments était plus forte pour les travailleurs des usines ayant connu le plus de licenciements [81]. Dans une enquête rétrospective parmi des salariés de Lombardie en Italie, une augmentation dans l'utilisation de médicaments psychotropes, en particulier d'antidépresseurs, a été trouvée mais cette augmentation ne déviait pas de la tendance observée avant la crise [82], ce qui ne correspondait pas à nos résultats.

En population générale, il a été montré que l'utilisation d'hypnotiques était restée stable entre 1983-1987 et 1992-1995 dans le sud de la Finlande où une crise économique est survenue au début des années 90 [78], ce qui concordait avec nos résultats observés en population générale.

\section{La santé perçue}

Quant à la santé perçue, nos résultats ont montré une augmentation de la prévalence de mauvaise santé perçue entre 2006 et 2010 dans la population au travail et parmi les hommes en population générale en France. A notre connaissance, aucune étude n'a été réalisée sur l'évolution de la mauvaise santé perçue dans la population au travail en période de crise économique. 
Dans la population générale, la littérature sur le sujet montrait des résultats mixtes. En désaccord avec nos résultats, une étude japonaise a observé une diminution de la prévalence de mauvaise santé perçue entre 1986-1989 et 1998-2001 malgré la crise économique des pays asiatiques en 1997 [83]. Cependant, en accord avec nos résultats, des études ont montré une augmentation de la prévalence de mauvaise santé perçue, notamment en Grèce entre avant et après le début de la crise économique de 2008 mais pas en Irlande [35,36]. En Estonie et en Lituanie, une stabilisation entre 2008 et 2010 après une tendance à la baisse entre 2004 et 2008 a été observée tandis qu'en Finlande la prévalence de mauvaise santé perçue était stable entre 2004 et 2010 [41]. Enfin, en Grèce, il a été montré que les individus en 2010 déclaraient plus fréquemment être en mauvaise santé que les individus interrogés en 2006 [37]. Ces dernières études étaient donc en accord, sauf pour la Finlande et l'Irlande, avec nos résultats montrant une augmentation de la prévalence de mauvaise santé perçue pendant la période de crise économique.

\section{Evolutions différentielles}

Nous avons également mis en évidence des évolutions différentielles de la consommation d'alcool à risque selon la profession chez les femmes, montrant que les professions intermédiaires et les employées étaient les plus à risque d'une augmentation de la prévalence de la consommation d'alcool à risque. A notre connaissance, une seule étude a exploré les évolutions différentielles de la consommation d'alcool et a observé une augmentation des symptômes de dépendance à l'alcool qui était plus forte pour les femmes les plus âgées entre 2000 et 2010 aux Etats-Unis [34].

D'après nos résultats, l'évolution de la prévalence des problèmes de sommeil et des troubles du sommeil différait selon l'origine parmi les femmes, une augmentation ayant été trouvée parmi les femmes d'origine étrangère mais pas parmi les femmes d'origine française. Quant à l'évolution de la prévalence des problèmes de sommeil et de la durée de 
sommeil insuffisante, elle différait selon l'âge et le type de contrat chez les hommes, les plus jeunes (20 à 40 ans) et les personnes en contrat permanent étaient les plus à risque d'une dégradation. L'évolution de la prise de psychotropes et d'antidépresseurs a aussi montré une évolution différente selon le secteur public/privé parmi les femmes, montrant une tendance à la dégradation dans le secteur public et à l'amélioration dans le secteur privé. Cependant, aucune autre étude, à notre connaissance, n'a étudié les évolutions différentielles des variables de sommeil, et de la prise de psychotropes en période de crise économique.

Nos résultats ont aussi montré des évolutions différentielles de la prévalence de mauvaise santé perçue selon l'âge (parmi les hommes seulement) et le type de contrat, en effet les hommes de 30 à 40 ans et de plus de 50 ans ainsi que les personnes en contrat permanent avaient plus de risque d'une dégradation de leur santé perçue que les autres entre 2006 et 2010 en France. Dans la littérature, des évolutions différentielles de la prévalence de mauvaise santé perçue ont également été montrées selon l'âge, la profession et le niveau d'éducation. En Estonie et en Lituanie, il y a été observé une augmentation de la prévalence de la mauvaise santé perçue chez les plus jeunes et une légère diminution chez les plus âgés entre 2008 et 2010, ce qui ne concordait pas avec nos résultats, et l'augmentation était plus forte pour les plus diplômés [41]. Au Japon, l'augmentation de la prévalence de la mauvaise santé perçue était plus marquée pour les employés que pour les autres professions, ce qui a renforcé les disparités de mauvaise santé perçue selon la profession durant la crise économique des années 90 [83].

\section{Forces et limites}

A notre connaissance, cette étude est la première étude prospective explorant l'évolution de la consommation d'alcool à risque, du tabagisme, et de la santé perçue et une des rares études à étudier l'évolution du sommeil et de la prise de psychotropes en période de crise 
économique à l'aide d'un échantillon national représentatif de la population au travail, et évaluant les évolutions différentielles selon des sous-groupes de population. De plus, les taux de réponse et de suivi étaient satisfaisants ( $76 \%$ et $81 \%)$. En outre, les pondérations ont été incluses dans toutes les analyses afin de prendre en compte le biais potentiel lié à la non-réponse en 2006 et à l'attrition en 2010, et de permettre la généralisation des résultats à la population au travail de 2006 en France.

Cependant, certaines limites doivent être mentionnées. Il pourrait y avoir un potentiel biais de sélection puisque 19\% de l'échantillon de 2006 n'a pas participé à l'enquête de 2010, ce sous-groupe pourrait ne pas avoir participé à cause d'une mauvaise santé mentale menant ainsi à une sous-estimation des résultats. Cependant, les pondérations ont été incluses afin de limiter ce biais. Un potentiel effet «travailleur sain » doit aussi être considéré puisque les individus exclus des analyses, parce qu'ils ne travaillaient plus en 2010, pourraient être plus susceptibles de souffrir d'une dégradation de leur santé mentale avant de quitter le marché du travail. Des analyses complémentaires ont montré que cet effet de sélection serait faible. Par conséquent, cela pourrait mener à une petite sous-estimation des résultats. De plus, notre étude était basée sur deux collectes de données séparées de quatre ans et ne peut donc pas refléter toutes les fluctuations de la prévalence des troubles mentaux au cours de cette période. Concernant le tabagisme, seul le statut tabagique a été étudié. Les résultats sur les problèmes de sommeil, étant une combinaison des troubles du sommeil et de la durée de sommeil insuffisante, étaient surtout influencés par l'augmentation de la prévalence de la durée insuffisante notamment chez les hommes. Des analyses de sensibilité réalisées avec des seuils différents pour le découpage des variables relatives aux psychotropes ont montré des résultats robustes, à savoir aucune évolution pour ces variables entre 2006 et 2010. A cause de la multiplicité des tests, nos résultats significatifs pourraient être dus au hasard. Cependant, certains auteurs ont suggéré que contrôler pour la 
multiplicité des tests ne serait pas nécessaire dans le cas d'études exploratoires [84]. De plus, l'étude présentant un caractère exploratoire, il peut être difficile d'interpréter les évolutions observées, notamment certaines évolutions différentielles. Toute tentative d'explication ne peut être que spéculative et il convient donc de garder une grande prudence dans l'interprétation, en particulier au regard du rôle causal de la crise.

\section{Conclusion}

En conclusion, les politiques de prévention devraient considérer que, malgré des prévalences de pathologies mentales stables à court terme, les prévalences des comportements et indicateurs de santé mentale peuvent avoir augmenté en période de crise économique. De plus, du fait d'une dégradation plus marquée, certains sous-groupes de la population, tels que les plus jeunes, pourraient être d'un intérêt particulier pour les politiques de prévention.

\section{Conflit d'intérêt : aucun}

Remerciements : les auteurs remercient le groupe de travail de la DREES et de la DARES pour leurs aide et conseils sur les données SIP. Les auteurs remercient également les membres de la DARES, de la DREES, du CEE et de l'INSEE, et toutes les personnes qui ont participé à l'enquête SIP et qui ont rendu cette étude possible.

Financements : cette étude a été financée par le Ministère du travail (DARES, subvention $\mathrm{n}^{\circ}$ 2200727156) et l'Université de Versailles Saint-Quentin-en-Yvelines (thèse de Lucile Malard). 


\section{Références}

[1] WHO. Health in times of global economic crisis: implications for the WHO European Region 2009. http://www.euro.who.int/en/health-topics/Health-systems/healthsystems-governance/publications/2009/health-in-times-of-global-economic-crisisimplications-for-the-who-european-region (accessed September 25, 2014).

[2] HCSP. Crise économique, santé et inégalités sociales de santé. Paris: Haut Conseil de la Santé Publique; 2016.

[3] Ruhm CJ. Recessions, Healthy No More? National Bureau of Economic Research; 2013.

[4] Ruhm CJ. Are Recessions Good for Your Health? The Quarterly Journal of Economics 2000;115:617-50. doi:10.1162/003355300554872.

[5] Evans W, Graham JD. Traffic safety and the business cycle. Alcohol, Drugs, and Driving 1988;4.

[6] Brenner $\mathrm{MH}$, Mooney A. Unemployment and health in the context of economic change. Soc Sci Med 1983;17:1125-38.

[7] Barr B, Taylor-Robinson D, Scott-Samuel A, McKee M, Stuckler D. Suicides associated with the 2008-10 economic recession in England: time trend analysis. BMJ 2012;345:e5142.

[8] Baumbach A, Gulis G. Impact of financial crisis on selected health outcomes in Europe. Eur J Public Health 2014;24:399-403. doi:10.1093/eurpub/cku042.

[9] Chan CH, Caine ED, You S, Fu KW, Chang SS, Yip PSF. Suicide rates among working-age adults in South Korea before and after the 2008 economic crisis. J Epidemiol Community Health 2014;68:246-52. doi:10.1136/jech-2013-202759.

[10] Chang S-S, Stuckler D, Yip P, Gunnell D. Impact of 2008 global economic crisis on suicide: time trend study in 54 countries. BMJ 2013;347:f5239.

[11] Chang S-S, Gunnell D, Sterne JAC, Lu T-H, Cheng ATA. Was the economic crisis 1997-1998 responsible for rising suicide rates in East/Southeast Asia? A time-trend analysis for Japan, Hong Kong, South Korea, Taiwan, Singapore and Thailand. Social Science \& Medicine 2009;68:1322-31. doi:10.1016/j.socscimed.2009.01.010.

[12] De Vogli R, Marmot M, Stuckler D. Excess suicides and attempted suicides in Italy attributable to the great recession. J Epidemiol Community Health 2013;67:378-9. doi:10.1136/jech-2012-201607.

[13] Falagas ME, Vouloumanou EK, Mavros MN, Karageorgopoulos DE. Economic crises and mortality: a review of the literature. Int J Clin Pract 2009;63:1128-35. doi:10.1111/j.1742-1241.2009.02124.x.

[14] Reeves A, Stuckler D, McKee M, Gunnell D, Chang S-S, Basu S. Increase in state suicide rates in the USA during economic recession. Lancet 2012;380:1813-4. doi:10.1016/S0140-6736(12)61910-2.

[15] Stuckler D, Basu S, Suhrcke M, Coutts A, McKee M. Effects of the 2008 recession on health: a first look at European data. Lancet 2011;378:124-5. doi:10.1016/S01406736(11)61079-9.

[16] Uutela A. Economic crisis and mental health. Curr Opin Psychiatry 2010;23:127-30. doi:10.1097/YCO.0b013e328336657d.

[17] Vlachadis N, Vlachadi M, Iliodromiti Z, Kornarou E, Vrachnis N. Greece's economic crisis and suicide rates: overview and outlook. J Epidemiol Community Health 2014;68:1204-5. doi:10.1136/jech-2014-204407.

[18] Haw C, Hawton K, Gunnell D, Platt S. Economic recession and suicidal behaviour: Possible mechanisms and ameliorating factors. Int J Soc Psychiatry 2015;61:73-81. doi:10.1177/0020764014536545. 
[19] Laanani M, Ghosn W, Jougla E, Rey G. Impact of unemployment variations on suicide mortality in Western European countries (2000-2010). J Epidemiol Community Health 2015;69:103-9. doi:10.1136/jech-2013-203624.

[20] Madianos M, Economou M, Alexiou T, Stefanis C. Depression and economic hardship across Greece in 2008 and 2009: two cross-sectional surveys nationwide. Soc Psychiatry Psychiatr Epidemiol 2011;46:943-52. doi:10.1007/s00127-010-02654.

[21] Gili M, Roca M, Basu S, McKee M, Stuckler D. The mental health risks of economic crisis in Spain: evidence from primary care centres, 2006 and 2010. Eur J Public Health 2013;23:103-8. doi:10.1093/eurpub/cks035.

[22] Van Hal G. The true cost of the economic crisis on psychological well-being: a review. Psychol Res Behav Manag 2015;8:17-25. doi:10.2147/PRBM.S44732.

[23] Catalano R, Goldman-Mellor S, Saxton K, Margerison-Zilko C, Subbaraman M, LeWinn K, et al. The health effects of economic decline. Annu Rev Public Health 2011;32:431-50. doi:10.1146/annurev-publhealth-031210-101146.

[24] Barr B, Kinderman P, Whitehead M. Trends in mental health inequalities in England during a period of recession, austerity and welfare reform 2004 to 2013 . Social Science \& Medicine 2015;147:324-31. doi:10.1016/j.socscimed.2015.11.009.

[25] Economou M, Madianos M, Peppou LE, Patelakis A, Stefanis CN. Major depression in the era of economic crisis: a replication of a cross-sectional study across Greece. $\mathrm{J}$ Affect Disord 2013;145:308-14. doi:10.1016/j.jad.2012.08.008.

[26] Fountoulakis KN, Savopoulos C, Siamouli M, Zaggelidou E, Mageiria S, Iacovides A, et al. Trends in suicidality amid the economic crisis in Greece. Eur Arch Psychiatry Clin Neurosci 2013;263:441-4. doi:10.1007/s00406-012-0385-9.

[27] Miret M, Caballero FF, Huerta-Ramírez R, Moneta MV, Olaya B, Chatterji S, et al. Factors associated with suicidal ideation and attempts in Spain for different age groups. Prevalence before and after the onset of the economic crisis. J Affect Disord 2014;163:1-9. doi:10.1016/j.jad.2014.03.045.

[28] Mattei G, Ferrari S, Pingani L, Rigatelli M. Short-term effects of the 2008 Great Recession on the health of the Italian population: an ecological study. Soc Psychiatry Psychiatr Epidemiol 2014;49:851-8. doi:10.1007/s00127-014-0818-z.

[29] Stuckler D, Basu S. The Body Economic: Why Austerity Kills. First Edition edition. New York: Basic Books; 2013.

[30] Stuckler D, Basu S, Suhrcke M, Coutts A, McKee M. The public health effect of economic crises and alternative policy responses in Europe: an empirical analysis. Lancet 2009;374:315-23. doi:10.1016/S0140-6736(09)61124-7.

[31] Mulia N, Zemore SE, Murphy R, Liu H, Catalano R. Economic loss and alcohol consumption and problems during the 2008 to 2009 U.S. recession. Alcohol Clin Exp Res 2014;38:1026-34. doi:10.1111/acer.12301.

[32] Murphy RD, Zemore SE, Mulia N. Housing instability and alcohol problems during the 2007-2009 US recession: the moderating role of perceived family support. J Urban Health 2014;91:17-32. doi:10.1007/s11524-013-9813-z.

[33] Vijayasiri G, Richman JA, Rospenda KM. The Great Recession, somatic symptomatology and alcohol use and abuse. Addict Behav 2012;37:1019-24. doi:10.1016/j.addbeh.2012.04.007.

[34] Zemore SE, Karriker-Jaffe KJ, Mulia N. Temporal Trends and Changing Racial/ethnic Disparities in Alcohol Problems: Results from the 2000 to 2010 National Alcohol Surveys. J Addict Res Ther 2013;4. doi:10.4172/21556105.1000160. 
[35] Hessel P, Vandoros S, Avendano M. The differential impact of the financial crisis on health in Ireland and Greece: a quasi-experimental approach. Public Health 2014;128:911-9. doi:10.1016/j.puhe.2014.08.004.

[36] Vandoros S, Hessel P, Leone T, Avendano M. Have health trends worsened in Greece as a result of the financial crisis? A quasi-experimental approach. Eur J Public Health 2013;23:727-31. doi:10.1093/eurpub/ckt020.

[37] Zavras D, Tsiantou V, Pavi E, Mylona K, Kyriopoulos J. Impact of economic crisis and other demographic and socio-economic factors on self-rated health in Greece. Eur J Public Health 2013;23:206-10. doi:10.1093/eurpub/cks143.

[38] Bor J, Basu S, Coutts A, McKee M, Stuckler D. Alcohol use during the great recession of 2008-2009. Alcohol Alcohol 2013;48:343-8. doi:10.1093/alcalc/agt002.

[39] Harhay MO, Bor J, Basu S, McKee M, Mindell JS, Shelton NJ, et al. Differential impact of the economic recession on alcohol use among white British adults, 20042010. Eur J Public Health 2014;24:410-5. doi:10.1093/eurpub/ckt134.

[40] Ásgeirsdóttir TL, Corman H, Noonan K, Ólafsdóttir P, Reichman NE. Was the economic crisis of 2008 good for Icelanders? Impact on health behaviors. Econ Hum Biol 2014;13:1-19. doi:10.1016/j.ehb.2013.03.005.

[41] Reile R, Helakorpi S, Klumbiene J, Tekkel M, Leinsalu M. The recent economic recession and self-rated health in Estonia, Lithuania and Finland: a comparative cross-sectional study in 2004-2010. J Epidemiol Community Health 2014;68:1072-9. doi:10.1136/jech-2014-204196.

[42] Birnbaum HG, Kessler RC, Kelley D, Ben-Hamadi R, Joish VN, Greenberg PE. Employer burden of mild, moderate, and severe major depressive disorder: mental health services utilization and costs, and work performance. Depress Anxiety 2010;27:78-89. doi:10.1002/da.20580.

[43] Kessler RC, Akiskal HS, Ames M, Birnbaum H, Greenberg P, Hirschfeld RMA, et al. Prevalence and effects of mood disorders on work performance in a nationally representative sample of U.S. workers. Am J Psychiatry 2006;163:1561-8. doi:10.1176/appi.ajp.163.9.1561.

[44] Malard L, Chastang J-F, Schütte S, Parent-Thirion A, Vermeylen G, Niedhammer I. Changes in psychosocial work exposures among employees between 2005 and 2010 in 30 countries in Europe. J Occup Environ Med 2013;55:1135-41. doi:10.1097/JOM.0b013e3182a3eb90.

[45] Malard L, Chastang J-F, Niedhammer I. Changes in psychosocial work factors in the French working population between 2006 and 2010. Int Arch Occup Environ Health 2015;88:235-46. doi:10.1007/s00420-014-0953-6.

[46] Nena E, Steiropoulos P, Papanas N, Kougkas D, Zarogoulidis P, Constantinidis T. Greek financial crisis: From loss of money to loss of sleep? Hippokratia 2014;18:135-8.

[47] Rostila M. The Swedish labour market in the 1990s: the very last of the healthy jobs? Scand J Public Health 2008;36:126-34. doi:10.1177/1403494807085067.

[48] Malard L, Chastang J-F, Niedhammer I. Changes in major depressive and generalized anxiety disorders in the national French working population between 2006 and 2010. J Affect Disord 2015;178:52-9. doi:10.1016/j.jad.2015.02.027.

[49] Doeringer P, Piore MJ. Internal labor markets and manpower adjustment. New York 1971.

[50] Doeringer PB, Piore MJ. Internal Labor Markets and Manpower Analysis. M.E. Sharpe; 1985.

[51] Cain GG. The Challenge of Segmented Labor Market Theories to Orthodox Theory: A Survey. Journal of Economic Literature 1976;14:1215-57. 
[52] Reich M, Gordon DM, Edwards RC. A Theory of Labor Market Segmentation. The American Economic Review 1973;63:359-65.

[53] Jaoul-Grammare M. L'évolution de la segmentation du marché du travail en France: 1973-2007. Bureau d'Economie Théorique et Appliquée, UDS, Strasbourg; 2011.

[54] Verick S. Who is Hit Hardest During a Financial Crisis? The Vulnerability of Young Men and Women to Unemployment in an Economic Downturn. Rochester, NY: Social Science Research Network; 2009.

[55] Vaughan-Whitehead D. Work Inequalities in the Crisis: Evidence from Europe. Edward Elgar Publishing; 2011.

[56] Burström B, Fredlund P. Self rated health: Is it as good a predictor of subsequent mortality among adults in lower as well as in higher social classes? J Epidemiol Community Health 2001;55:836-40.

[57] Idler EL, Benyamini Y. Self-rated health and mortality: a review of twenty-seven community studies. J Health Soc Behav 1997;38:21-37.

[58] Månsson NO, Råstam L. Self-rated health as a predictor of disability pension and death--a prospective study of middle-aged men. Scand J Public Health 2001;29:1518.

[59] Mavaddat N, Kinmonth AL, Sanderson S, Surtees P, Bingham S, Khaw KT. What determines Self-Rated Health (SRH)? A cross-sectional study of SF-36 health domains in the EPIC-Norfolk cohort. J Epidemiol Community Health 2011;65:800-6. doi:10.1136/jech.2009.090845.

[60] Molarius A, Janson S. Self-rated health, chronic diseases, and symptoms among middle-aged and elderly men and women. J Clin Epidemiol 2002;55:364-70.

[61] Singh-Manoux A, Martikainen P, Ferrie J, Zins M, Marmot M, Goldberg M. What does self rated health measure? Results from the British Whitehall II and French Gazel cohort studies. J Epidemiol Community Health 2006;60:364-72. doi:10.1136/jech.2005.039883.

[62] Coutrot T, Rouxel C, Bahu M, Herbet J-B, Mermilliod C. Parcours professionnels et état de santé. Premières Informations et Premières Synthèses 2010;1.

[63] Chazelle E, Chastang J-F, Niedhammer I. Psychosocial work factors and sleep problems: findings from the French national SIP survey. Int Arch Occup Environ Health 2015. doi:10.1007/s00420-015-1087-1.

[64] Lassalle M, Chastang J-F, Niedhammer I. Working conditions and psychotropic drug use: cross-sectional and prospective results from the French national SIP study. J Psychiatr Res 2015;63:50-7. doi:10.1016/j.jpsychires.2015.02.019.

[65] Murcia M, Chastang J-F, Niedhammer I. Educational inequalities in major depressive and generalized anxiety disorders: results from the French national SIP study. Soc Psychiatry Psychiatr Epidemiol 2015;50:919-28. doi:10.1007/s00127-015-1010-9.

[66] Murcia M, Chastang J-F, Niedhammer I. Psychosocial work factors, major depressive and generalised anxiety disorders: results from the French national SIP study. J Affect Disord 2013;146:319-27. doi:10.1016/j.jad.2012.09.014.

[67] Niedhammer I, Malard L, Chastang J-F. Occupational factors and subsequent major depressive and generalized anxiety disorders in the prospective French national SIP study. BMC Public Health 2015;15:200. doi:10.1186/s12889-015-1559-y.

[68] Niedhammer I, Chastang J-F. Psychosocial work factors and first depressive episode: retrospective results from the French national SIP survey. International Archives of Occupational and Environmental Health 2014.

[69] De Riccardis N. Traitements de la non-réponse et calages pour l'enquête Santé et Itinéraire Professionnel de 2010. Document de Travail, Série Sources et Méthodes 2012;36. 
[70] Hardin JW, Hilbe JM. Generalized Estimating Equations, Second Edition. CRC Press; 2012.

[71] Liang K-Y, Zeger SL. Longitudinal data analysis using generalized linear models. Biometrika 1986;73:13-22. doi:10.1093/biomet/73.1.13.

[72] Hessel P, Vandoros S, Avendano M. The differential impact of the financial crisis on health in Ireland and Greece: a quasi-experimental approach. Public Health 2014;128:911-9. doi:10.1016/j.puhe.2014.08.004.

[73] Harper S, Charters TJ, Strumpf EC, Galea S, Nandi A. Economic downturns and suicide mortality in the USA, 1980-2010: observational study. Int J Epidemiol 2015;44:956-66. doi:10.1093/ije/dyv009.

[74] Gasparrini A, Lopez Bernal J. Commentary: On the use of quasi-experimental designs in public health evaluation. Int $\mathbf{J}$ Epidemiol 2015;44:966-8. doi:10.1093/ije/dyv065.

[75] Beck F, Guignard R, Richard J-B, Wilquin J-L, Pererri-Watel P. Augmentation récente du tabagisme en France: principaux résultats du Baromètre santé, France, 2010. BEH 2011:230-3.

[76] Beck F, Richard J-B. [Alcohol use in France]. Presse Med 2014;43:1067-79. doi:10.1016/j.lpm.2014.02.027.

[77] Dávalos ME, Fang H, French MT. Easing the pain of an economic downturn: macroeconomic conditions and excessive alcohol consumption. Health Econ 2012;21:1318-35. doi:10.1002/hec.1788.

[78] Hyyppä MT, Kronholm E, Alanen E. Quality of sleep during economic recession in Finland: a longitudinal cohort study. Soc Sci Med 1997;45:731-8.

[79] Pacula RL. Substance use and recessions: what can be learned from economic analyses of alcohol? Int J Drug Policy 2011;22:326-34. doi:10.1016/j.drugpo.2011.07.008.

[80] Dregan A, Armstrong D. Age, cohort and period effects in the prevalence of sleep disturbances among older people: the impact of economic downturn. Soc Sci Med 2009;69:1432-8. doi:10.1016/j.socscimed.2009.08.041.

[81] Modrek S, Hamad R, Cullen MR. Psychological well-being during the great recession: changes in mental health care utilization in an occupational cohort. Am J Public Health 2015;105:304-10. doi:10.2105/AJPH.2014.302219.

[82] Vittadini G, Beghi M, Mezzanzanica M, Ronzoni G, Cornaggia CM. Use of psychotropic drugs in Lombardy in time of economic crisis (2007-2011): A population-based study of adult employees. Psychiatry Res 2014. doi:10.1016/j.psychres.2014.06.006.

[83] Kondo N, Subramanian SV, Kawachi I, Takeda Y, Yamagata Z. Economic recession and health inequalities in Japan: analysis with a national sample, 1986-2001. J Epidemiol Community Health 2008;62:869-75. doi:10.1136/jech.2007.070334.

[84] Bender R, Lange S. Adjusting for multiple testing--when and how? J Clin Epidemiol 2001;54:343-9. 
Tableau 1 Description de l'échantillon d'étude

\begin{tabular}{|c|c|c|c|c|c|c|c|c|c|c|c|}
\hline & & \multicolumn{4}{|c|}{ Hommes $(\mathrm{N}=2679)$} & \multicolumn{4}{|c|}{ Femmes $(\mathrm{N}=2921)$} & \multirow[t]{3}{*}{$\mathrm{p}_{2006^{\mathrm{c}}}$} & \multirow[t]{3}{*}{$\mathrm{p}_{2010}{ }^{\mathrm{d}}$} \\
\hline & & \multicolumn{2}{|c|}{2006} & \multicolumn{2}{|c|}{2010} & \multicolumn{2}{|c|}{2006} & \multicolumn{2}{|c|}{2010} & & \\
\hline & & $\mathrm{N}^{\mathrm{a}}$ & $\%^{\mathrm{b}}$ & $\mathrm{N}^{\mathrm{a}}$ & $\%^{\mathrm{b}}$ & $\mathrm{N}^{\mathrm{a}}$ & $\%^{\mathrm{b}}$ & $\mathrm{N}^{\mathrm{a}}$ & $\%^{\mathrm{b}}$ & & \\
\hline \multirow[t]{4}{*}{ Age (années) } & {$[20 ; 30[$} & 365 & 20,52 & 167 & 9,64 & 372 & 19,79 & 182 & 9,72 & ns & ns \\
\hline & {$[30 ; 40[$} & 778 & 31,01 & 601 & 27,94 & 826 & 28,6 & 640 & 26,49 & & \\
\hline & {$[40 ; 50[$} & 916 & 31,58 & 938 & 33,09 & 1061 & 32,28 & 1012 & 31,35 & & \\
\hline & $50+$ & 620 & 16,89 & 973 & 29,32 & 662 & 19,33 & 1087 & 32,44 & & \\
\hline \multirow[t]{2}{*}{ Origine } & Française & 2479 & 91,68 & 2479 & 91,68 & 2726 & 92,19 & 2726 & 92,19 & ns & ns \\
\hline & Etrangère & 200 & 8,32 & 200 & 8,32 & 195 & 7,81 & 195 & 7,81 & & \\
\hline \multirow[t]{4}{*}{ Profession } & Cadres & 458 & 17,14 & 534 & 20,13 & 379 & 11,42 & 437 & 13,15 & $* * *$ & $* * *$ \\
\hline & $\begin{array}{l}\text { Professions } \\
\text { intermédiaires }\end{array}$ & 706 & 26,94 & 743 & 28,29 & 814 & 26,55 & 857 & 28,62 & & \\
\hline & Employés & 536 & 22,44 & 536 & 20,28 & 1488 & 52,26 & 1432 & 50,09 & & \\
\hline & Ouvriers & 973 & 33,48 & 865 & 31,3 & 227 & 9,77 & 195 & 8,14 & & \\
\hline \multirow[t]{4}{*}{ Secteur d'activité } & Agriculture et pêche & 191 & 5,81 & 183 & 5,70 & 73 & 1,81 & 65 & 1,69 & $* * *$ & $* * *$ \\
\hline & Industrie & 669 & 22,75 & 645 & 21,99 & 256 & 9,24 & 231 & 7,85 & & \\
\hline & Construction & 374 & 10,24 & 357 & 10,54 & 54 & 1,51 & 61 & 1,98 & & \\
\hline & Services & 1379 & 61,20 & 1479 & 61,78 & 2445 & 87,44 & 2540 & 88,47 & & \\
\hline \multirow[t]{2}{*}{ Secteur } & Public & 503 & 18,63 & 485 & 18,11 & 956 & 30,04 & 968 & 30,16 & $* * *$ & $* * *$ \\
\hline & Privé & 2127 & 81,37 & 2194 & 81,89 & 1886 & 69,96 & 1953 & 69,84 & & \\
\hline \multirow[t]{2}{*}{ Statut } & Indépendant & 296 & 10,88 & 362 & 12,65 & 214 & 7,25 & 233 & 7,74 & $* * *$ & $* * *$ \\
\hline & Salarié & 2334 & 89,12 & 2317 & 87,35 & 2628 & 92,75 & 2688 & 92,26 & & \\
\hline \multirow[t]{2}{*}{ Type de contrat } & Temporaire & 286 & 12,01 & 111 & 5,11 & 407 & 16,29 & 212 & 8,31 & $* * *$ & $* * *$ \\
\hline & Permanent & 2317 & 87,99 & 2463 & 94,89 & 2442 & 83,71 & 2660 & 91,69 & & \\
\hline Statut tabagique & Fumeur & 805 & 33,78 & 900 & 35,93 & 709 & 28,65 & 813 & 30,32 & $* *$ & $* * *$ \\
\hline
\end{tabular}




\begin{tabular}{|c|c|c|c|c|c|c|c|c|c|c|c|}
\hline \multirow{3}{*}{$\begin{array}{l}\text { Consommation } \\
\text { d'alcool à risque }\end{array}$} & Non-fumeur & 1636 & 66,22 & 1663 & 64,07 & 1964 & 71,35 & 2025 & 69,68 & \multirow{3}{*}{$* * *$} & \multirow[b]{2}{*}{$* *:$} \\
\hline & Oui & 1175 & 48,48 & 1196 & 47,25 & 393 & 15,95 & 494 & 18,74 & & \\
\hline & Non & 1219 & 51,52 & 1359 & 52,75 & 2219 & 84,05 & 2340 & 81,26 & & \\
\hline \multirow[t]{2}{*}{ Problèmes de sommeil } & Oui & 751 & 28,21 & 793 & 30,79 & 984 & 32,76 & 1058 & 36,20 & \multirow[t]{2}{*}{$* *$} & \multirow[t]{2}{*}{$* * *$} \\
\hline & Non & 1928 & 71,79 & 1886 & 69,21 & 1937 & 67,24 & 1863 & 63,80 & & \\
\hline \multirow[t]{2}{*}{ Troubles du sommeil } & Oui & 566 & 22,01 & 634 & 25,56 & 775 & 25,67 & 853 & 29,56 & \multirow[t]{2}{*}{$* * *$} & \multirow[t]{2}{*}{$* * *$} \\
\hline & Non & 2113 & 77,99 & 2045 & 74,44 & 2146 & 74,33 & 2068 & 70,44 & & \\
\hline \multirow[t]{2}{*}{$\begin{array}{l}\text { Durée de sommeil } \\
\text { insuffisante }\end{array}$} & Oui & 491 & 18,31 & 545 & 20,41 & 733 & 24,17 & 815 & 27,48 & \multirow[t]{2}{*}{$* *$} & \multirow[t]{2}{*}{$* *$} \\
\hline & Non & 2188 & 81,69 & 2134 & 79,59 & 2188 & 75,83 & 2106 & 72,52 & & \\
\hline \multirow[t]{2}{*}{ Prise de psychotropes } & Oui & 209 & 7,73 & 227 & 8,45 & 463 & 15,29 & 489 & 15,98 & \multirow[t]{2}{*}{$* * *$} & \multirow[t]{2}{*}{$* * *$} \\
\hline & Non & 2470 & 92,27 & 2452 & 91,55 & 2458 & 84,71 & 2432 & 84,02 & & \\
\hline \multirow[t]{2}{*}{ Prise d'antidépresseurs } & Oui & 104 & 3,92 & 127 & 4,75 & 277 & 8,96 & 294 & 9,50 & \multirow[t]{2}{*}{$* * *$} & \multirow[t]{2}{*}{$* * *$} \\
\hline & Non & 2575 & 96,08 & 2552 & 95,25 & 2644 & 91,04 & 2627 & 90,50 & & \\
\hline \multirow[t]{2}{*}{ Prise d'hypnotiques } & Oui & 74 & 2,58 & 73 & 2,68 & 121 & 3,74 & 145 & 4,63 & \multirow[t]{2}{*}{$*$} & \multirow[t]{2}{*}{$* *$} \\
\hline & Non & 2605 & 97,42 & 2606 & 97,32 & 2800 & 96,26 & 2776 & 95,37 & & \\
\hline \multirow[t]{2}{*}{ Prise d'anxiolytiques } & Oui & 106 & 4,16 & 113 & 3,98 & 226 & 7,60 & 253 & 8,30 & \multirow[t]{2}{*}{$* * *$} & \multirow[t]{2}{*}{$* * *$} \\
\hline & Non & 2573 & 95,84 & 2566 & 96,02 & 2695 & 92,40 & 2668 & 91,70 & & \\
\hline \multirow[t]{2}{*}{ Santé perçue } & Mauvaise & 437 & 15,29 & 581 & 20,98 & 543 & 19,44 & 699 & 24,50 & \multirow[t]{2}{*}{$* * *$} & \multirow[t]{2}{*}{$* *$} \\
\hline & Bonne & 2242 & 84,71 & 2098 & 79,02 & 2378 & 80,56 & 2222 & 75,50 & & \\
\hline
\end{tabular}

${ }^{\mathrm{a}}$ Nombre d'individus non-pondéré

${ }^{\mathrm{b}}$ Fréquence pondérée

${ }^{\mathrm{c}}$ Chi2 de Rao-Scott pour la différence entre hommes et femmes en 2006

${ }^{d}$ Chi2 de Rao-Scott pour la différence entre hommes et femmes en 2010

ns : p $>0,05 ; * \mathrm{p}<0,05 ; * * \mathrm{p}<0,01 ; * * * \mathrm{p}<0,001$ 
Tableau 2 Evolution de la prévalence des comportements et indicateurs de santé mentale en 2010 comparé à 2006 dans la population au travail en France : résultats de modèles d'équations d'estimation généralisées pondérés et ajustés sur l'âge

\begin{tabular}{|c|c|c|c|c|c|c|}
\hline \multirow[b]{2}{*}{ Année 2010 vs 2006} & \multicolumn{3}{|c|}{ Hommes } & \multicolumn{3}{|c|}{ Femmes } \\
\hline & OR & IC $95 \%$ & $\mathrm{p}$ & OR & IC $95 \%$ & $\mathrm{p}$ \\
\hline Tabagisme & 1,22 & {$[1,12 ; 1,32]$} & $* * *$ & 1,15 & {$[1,06 ; 1,25]$} & $* * *$ \\
\hline Consommation d'alcool à risque & 1,04 & {$[0,94 ; 1,16]$} & ns & 1,44 & {$[1,25 ; 1,65]$} & $* * *$ \\
\hline Problèmes de sommeil & 1,21 & {$[1,07 ; 1,35]$} & $* *$ & 1,10 & {$[0,98 ; 1,24]$} & ns \\
\hline Troubles du sommeil & 1,12 & {$[0,98 ; 1,28]$} & ns & 1,06 & {$[0,94 ; 1,20]$} & ns \\
\hline Durée de sommeil insuffisante & 1,33 & {$[1,17 ; 1,52]$} & $* * *$ & 1,18 & {$[1,04 ; 1,33]$} & $* *$ \\
\hline Prise de psychotropes & 1,03 & {$[0,83 ; 1,28]$} & ns & 0,94 & {$[0,82 ; 1,09]$} & ns \\
\hline Prise d'antidépresseurs & 1,16 & {$[0,87 ; 1,54]$} & ns & 0,96 & {$[0,81 ; 1,13]$} & ns \\
\hline Prise d'hypnotiques & 0,94 & {$[0,60 ; 1,46]$} & $\mathrm{ns}$ & 1,10 & {$[0,84 ; 1,44]$} & ns \\
\hline Prise d'anxiolytiques & 0,89 & {$[0,65 ; 1,24]$} & ns & 1,02 & {$[0,83 ; 1,25]$} & $\mathrm{ns}$ \\
\hline Mauvaise santé perçue & 1,29 & {$[1,12 ; 1,49]$} & $* * *$ & 1,19 & {$[1,04 ; 1,35]$} & $* *$ \\
\hline
\end{tabular}

ns : $\mathrm{p}>0,05 ; * \mathrm{p}<0,05 ; * * \mathrm{p}<0,01 ; * * * \mathrm{p}<0,001$ 
Tableau 3 Evolutions différentielles des comportements et indicateurs de santé mentale selon l'âge, l'origine, la profession, le secteur d'activité, le secteur public/privé, le statut indépendant/salarié et le type de contrat dans la population au travail en France entre 2006 et 2010

\begin{tabular}{|c|c|c|c|c|c|c|c|}
\hline \multirow{2}{*}{ Année 2010 vs 2006} & & \multicolumn{3}{|c|}{ Hommes } & \multicolumn{3}{|c|}{ Femmes } \\
\hline & & OR & IC 95\% & $\mathrm{p}$ & OR & IC 95\% & $\mathrm{p}$ \\
\hline Consommation d'alcool à & Profession & & & & & & $* \mathrm{a}$ \\
\hline \multirow[t]{4}{*}{ risque } & Cadres & & & & 1,04 & {$[0,74 ; 1,44]$} & $\mathrm{ns}$ \\
\hline & Professions intermédiaires & & & & 1,92 & {$[1,51 ; 2,44]$} & $* * *$ \\
\hline & Employés & & & & 1,29 & {$[1,04 ; 1,60]$} & $*$ \\
\hline & Ouvriers & & & & 1,38 & {$[0,74 ; 2,55]$} & ns \\
\hline \multirow[t]{11}{*}{ Problèmes de sommeil } & Age (années) & & & $* \mathbf{a}$ & & & \\
\hline & $20-30$ & $\mathbf{1 , 5 0}$ & {$[1,05 ; 2,13]$} & $*$ & & & \\
\hline & $30-40$ & 1,49 & {$[1,17 ; 1,89]$} & $* *$ & & & \\
\hline & $40-50$ & 0,96 & {$[0,79 ; 1,18]$} & ns & & & \\
\hline & $50+$ & 1,03 & {$[0,83 ; 1,26]$} & ns & & & \\
\hline & Origine & & & & & & $* \mathbf{a}$ \\
\hline & Français & & & & 1,05 & {$[0,94 ; 1,18]$} & ns \\
\hline & Etranger & & & & 1,92 & {$[1,21 ; 3,04]$} & $* *$ \\
\hline & Type de contrat & & & $* \mathbf{a}$ & & & \\
\hline & Temporaire & 0,61 & {$[0,36 ; 1,04]$} & ns & & & \\
\hline & Permanent & 1,23 & {$[1,09 ; 1,39]$} & $* *$ & & & \\
\hline \multirow[t]{3}{*}{ Troubles du sommeil } & Origine & & & & & & $* * \mathbf{a}$ \\
\hline & Français & & & & 1,00 & {$[0,88 ; 1,13]$} & ns \\
\hline & Etranger & & & & 2,28 & {$[1,35 ; 3,87]$} & $* *$ \\
\hline \multirow[t]{2}{*}{ Durée de sommeil insuffisante } & Age (années) & & & $* \mathbf{a}$ & & & \\
\hline & $20-30$ & 1,60 & {$[1,08 ; 2,37]$} & $*$ & & & \\
\hline
\end{tabular}




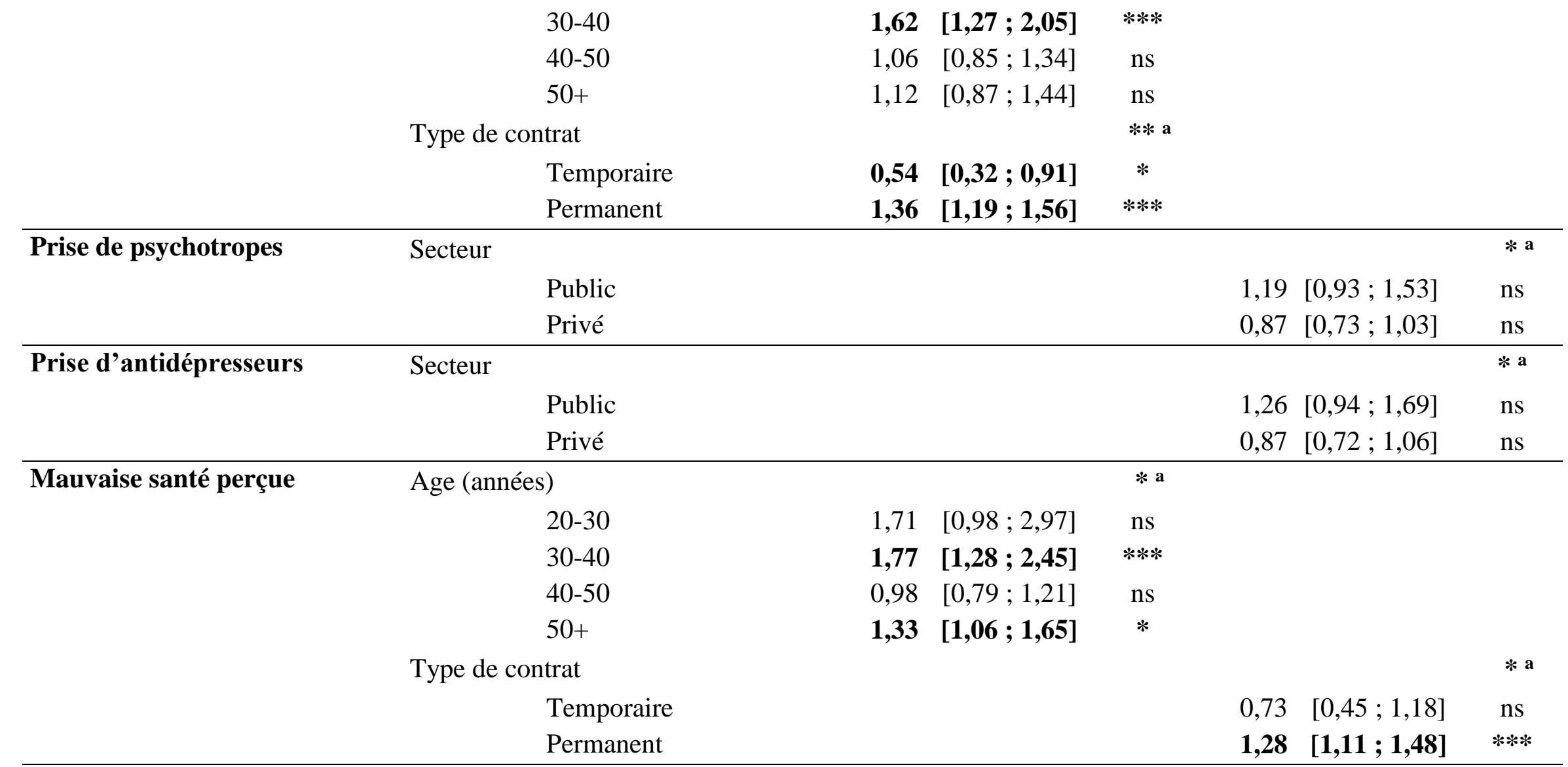

Seules les interactions significatives sont présentées

a significativité du terme d'interaction entre l'année et la covariable

OR pour chaque sous-groupe (référence 2006) ajusté sur l'âge

ns : $\mathrm{p}>0,05 ; * \mathrm{p}<0,05 ; * * \mathrm{p}<0,01 ; * * * \mathrm{p}<0,001$ 